\title{
Verbal Memory and IQ Predict Adaptive Behavior in Children and Adolescents with High-Functioning Autism Spectrum Disorders
}

\author{
O. Puig, ${ }^{a, b,{ }^{*}}$, R. Calvo ${ }^{a, b}$, M. Rosa ${ }^{a}$, E. de la Serna ${ }^{a, b}$, S. Lera-Miguel ${ }^{a}$, V. Sánchez-Gistau ${ }^{a, b}$ \\ and J. Castro-Fornieles ${ }^{\mathrm{a}, \mathrm{b}, \mathrm{c}, \mathrm{d}}$
}

aDepartment of Child and Adolescent Psychiatry and Psychology, SGR 1119. Hospital Clínic of Barcelona,
Spain
${ }^{b}$ Centro de Investigación Biomédica en Red de Salud Mental, CIBERSAM, Spain
${ }^{c}$ Institut d'Investigació Biomèdica August Pi i Sunyer, IDIBAPS, Barcelona, Spain
${ }^{d}$ Department of Psychiatry and Clinical Psychobiology, University of Barcelona, Spain

\begin{abstract}
Adaptive deficits are commonly found in high functioning autism spectrum disorders (HF-ASD) despite of cognitive potential. Most studies have focused on the relationships between adaptive behavior and intellectual quotient (IQ) and have used correlations to study relationships between them. Few studies have analyzed cognitive variables other than IQ as potential predictors of adaptive behavior in HF-ASD using regression methods. This study aimed to analyze the impact of several cognitive variables on adaptive behavior in a sample of children and adolescents with HFASD. METHODS: Sample included 16 child and adolescent boys with HF-ASD (age between 7-17 years). Cognitive assessment included measures of general intelligence, visual memory, verbal memory, working memory and problem solving/flexibility tests. Vineland Adaptive Behavior Scales (VABS) was used to evaluate adaptive behavior. To establish the predictive capacity of the cognitive variables for adaptive functioning, linear regression models were fitted for each adaptive domain using a stepwise method. RESULTS: Verbal memory and IQ emerged as the main independent predictors for VABS adaptive scores. The $41 \%$ of the variance in Communication was predicted by IQ. The $35 \%$ of the variance in Daily Living Skills was predicted by verbal memory. Almost half of the variance in Socialization was predicted by both, verbal memory and IQ (49\%). No other cognitive functions were associated with adaptive scores. CONCLUSIONS: The results highlight the strong impact of IQ and verbal memory on adaptive behavior in HF-ASD patients. These findings could contribute to identify potential targets of intervention.
\end{abstract}

Keywords: High-functioning autism spectrum disorders, adaptive behavior, cognition, verbal memory, IQ.

\section{INTRODUCTION}

High functioning autism spectrum disorders (HFASD) are associated with adaptive deficits, which have been consistently found to be lower than intellectual abilities, highlighting the magnitude of adaptive impairments despite cognitive potential in patients with HF-ASD [1-6]. Adaptive behavior is defined by the extent to which a person is capable of being selfsufficient in real-life situations, including the functional use of communication, socialization and daily living skills [7]. Clearly, improving adaptive skills is a meaningful and valued outcome of any intervention in autism. Thus, the identification of those variables that could predict adaptive behavior improvement emerges as an important issue. Cognitive abilities are known to be a functionally relevant feature of the disorder [1-8]. Therefore, identifying those cognitive impairments that are most closely associated with problematic behaviors and adaptive skills could open the route for the

\footnotetext{
*Address correspondence to this author at the Child and Adolescent Psychiatry and Psychology Department, Hospital Clinic of Barcelona. Villarroel 170, Barcelona, 08036, Spain; Tel/Fax: + 3493227 9974;

E-mail: opuig@clinic.ub.es
}

development of novel cognitive training approaches to treatment [8].

Intellectual quotient (IQ) has been the most studied variable among studies focusing on the relationships between adaptive behavior and cognitive characteristics $[1,3,4,6,9,10]$. These studies have demonstrated that IQ is positively correlated with and predicts adaptive behavior in both, low and highfunctioning autism. But in HF-ASD, the gap between IQ and adaptive impairments increases compared with lower functioning individuals $[3,6]$. Fewer studies have focused on other cognitive functions. Oliveras-Rentas et al. [4] found that working memory and processing speed, was significantly correlated with the Communication domain of the Vineland Adaptive Behavior Scales (VABS) [7]. Chang et al. [5] also found a significant relationship between working memory and the general adaptive composite score of the Adaptive Behavior Assessment System-II (ABAS-II) [11]. Gilotty et al. [12] found that the initiate and working memory domains of the metacognition index of the Behavior Rating Inventory for Executive Function (BRIEF) [13] questionnaire correlated with most domains of adaptive 
behavior. In one of the largest studies examining the relationships between several cognitive functions and adaptive skills, Ozonoff et al. [14] found that performance on a planning task was negatively associated with adaptive behavior.

Most of these studies have used correlations to examine the relationships between cognitive functions and adaptive scores. This approach informs us about the potential relations between the variables but does not allow us to know about the specific role of each of the variables as a predictor when controlling for the influence of the others, which could be addressed through regression models. Chang et al. [5] applied models of regression to examine the potential predictors of adaptive scores but only the full-scale IQ score was analysed as a predictor and the potential role of specific cognitive domains was not examined. Gilotty et al. [12] carried out regression models to explore both the role of $I Q$ and of the BRIEF'S metacognition index in the prediction of the VABS domains. They found that the metacognition index significantly predicted the socialization and communication domains of the VABS independently of the IQ. However, neurocognitive tests were not administered to assess cognition but the BRIEF questionnaire. Finally, Liss et al. [15] also used a regression approach to explore the unique contribution of specific cognitive domains to adaptive functioning. These authors found that the relationship between executive function and adaptive functioning was not significant when controlling for IQ. Recently, it has been suggested that the executive functions differences among individuals with autism would play a significant part in their real-life accomplishment, including everyday adaptive behavior [16] but, up to now, the specific value of executive functioning as a predictor of adaptive behavior remains unclear.

The role of other cognitive variables, such as visual and verbal memory, has deserved less study. Only Liss et al. [2] focused on the impact of memory on adaptive behavior. They found that in the HF-ASD group verbal memory was a main predictor for all domains of the VABS and language tests were significant predictors for the Communication domain. The authors concluded that verbal memory played a main role in adaptive functioning, but they also hypothesized other cognitive functions, which were not included in their study as working memory, would play an important role.

Charman et al. [8] highlighted the importance of studying cognitive abilities of individuals with autism in several cognitive domains, instead of examining only one or two cognitive functions. However, there is a scarcity of studies focusing in cognitive functions other than IQ as predictors of adaptive functioning in HFASD. As reviewed before, the predictive value of verbal memory and executive functions on adaptive behavior has not been extensively studied. Furthermore, although the visual processing deficits in autism have been described [17], no previous studies have focused on other cognitive skills as visual memory as potential adaptive predictors. The main objective of this study was to analyze the impact of several cognitive variables on adaptive functioning in a group of child and adolescents with HF-ASD. We included IQ as a potential predictor of adaptive functioning but also considered others variables much less studied such executive functions, memory and working memory.

\section{METHOD}

\subsection{Participants}

Participants were 16 children and adolescents with HF-ASD. Patients were recruited from the population referred to the Child and Adolescent Psychiatry and Psychology Department of Hospital Clinic in Barcelona, Spain. Inclusion criteria were age between 7 and 17 years and a primary diagnosis of DSM-IV-TR [18] ASD. Exclusion criteria were IQ below 70 , schizophrenia or bipolar disorder comorbidity, neurological disorders and language disability that could interfere with cognitive assessments. Two independent child and adolescent clinicians based on clinical interviews with patients and their families and using DSM-IV-TR criteria made diagnoses. All subjects were school aged and were boys. Age ranged between 7 and 15 years. Eight patients $(50 \%)$ had attention deficit symptoms corrected with stimulants. One patient $(6 \%)$ was on benzodiazepine because of sleep problems and another subject $(6 \%)$ was taking a combination of an antidepressant and an antipsychotic due to depressive symptoms and disruptive behavior (for more details see Table 1). The Review Boards of the institution approved the study and all parents gave written informed consent and subjects agreed to participate.

\subsection{Cognitive Measures}

A clinical psychologist with experience in child and adolescent cognitive assessment administered neurocognitive testing. To provide a standard metric for comparisons across cognitive variables and control for developmental stage, tests were converted to 
Table 1: Sample Characteristics

\begin{tabular}{|c|c|c|}
\hline & Mean & SD \\
\hline Male/female (n) & $16 / 0$ & \\
\hline Age & 11.13 & 2.36 \\
\hline \multicolumn{3}{|l|}{ Cognitive measures $^{a}$} \\
\hline IQ & 48.06 & 7.28 \\
\hline Verbal Memory & 48.63 & 7.79 \\
\hline Working Memory & 49.33 & 11.46 \\
\hline Problem Solving & 50.75 & 9.53 \\
\hline Visual Memory & 44.00 & 13.69 \\
\hline \multicolumn{3}{|l|}{ ADAPTIVE DOMAINS ${ }^{b}$} \\
\hline Communication & 87.13 & 13.23 \\
\hline Daily Living Skills & 84.56 & 12.07 \\
\hline Socialization & 80.31 & 18.82 \\
\hline
\end{tabular}

${ }^{a}$ Cognitive measures: $T$ scores $(M=50, S D=10)$.

${ }^{\mathrm{b}}$ Adaptive domains: Standard scores $(\mathrm{M}=100, \mathrm{SD}=15)$.

demographically corrected T-scores $(M=50, S D=10)$. Cognitive domains were derived in order to reduce the number of variables. The domains score represented the average of the T-scores for the tests included in the domain, if more than one:

IQ: Vocabulary and Matrix Reasoning subtests of the Spanish version of Wechsler Intelligence scales for children (WISC-IV). We used these two subtests as a proxy for IQ because they are the most high correlated subtests with the total IQ score [19].

Verbal memory: immediate and delayed recall of Memory of Histories subtests from the TOMAL battery [20].

- Visual memory: copy and immediate recall subtest of the Rey-Osterrich Complex Figure Test (ROCFT) [21].

Working memory: WISC-IV Letter-Number Sequencing subtest.

Problem solving/flexibility: total errors and total perseverative errors of the Wisconsin Card Sorting Test (WCST) [22].

\subsection{Adaptive Behavior Measure}

The Vineland Adaptive Behavior Scales $2^{\text {nd }}$ edition - Parent/Caregiver Rating Form [7] was used to assess adaptive functioning. The VABS is a standardized instrument that measures adaptive behavior in differentiated domains: Communication, Daily living skills and Socialization. Standard scores $(M=100, S D$ $=15$ ) are provided for each domain, with higher scores indicating more mature adaptive skills in all domains. The VABS has good psychometric properties with testretest reliability coefficients between 0.88 and 0.92 , and inter-rater reliability coefficients ranging from 0.70 to 0.80 [7].

\section{RESULTS}

\subsection{Results on Cognitive and Adaptive Functioning}

Statistical conditions for parametric statistics were checked and applied for all analyses. All tests were two-tailed (Statistical Package for the Social Sciences, v. 18.0). Table 1 shows sample characteristics. VABS scores indicate mild adaptive dysfunction for HF-ASD patients in all domains and neurocognitive scores were in the low-average range in almost all domains.

\subsection{Associations Between Adaptive Functioning and Cognitive Domains and Predictive Value of Cognitive Variables on Adaptive Functioning}

To study the relationship between adaptive functioning and cognitive scores, correlation coefficients were obtained using Pearson correlations. IQ showed significant correlations with the three VABS domains scores (Communication: $r=0.67, p=0.005$; Daily living skills: $r=0.59, p=0.017$; Socialization: $r=$ $0.62, p=0.011$ ). Verbal memory was significantly correlated with the Daily living skills $(r=0.63, p=$ $0.010)$ and Socialization domains $(r=0.62, p=0.010)$. The other cognitive variables were not significantly correlated with VABS domains.

To establish the predictive capacity of the cognitive variables for adaptive functioning, linear regression models were fitted for each adaptive domain using a stepwise method. Those variables which showed a significant correlation value with a particular VABS domain score were included in the regression models in the same block as potential predictors for that adaptive domain. The Communication domain was uniquely predicted by IQ, which explained $41 \%$ of variance (Table 2). For the Daily Living Skills domain, verbal memory emerged as the main predictor explaining $35 \%$ of the variance. $I Q(t=1.962, p=$ 0.072 ) did not explain a significant amount of variance beyond that accounted for by verbal memory. For the Socialization domain, verbal memory and IQ explained $49 \%$ of the variance. Verbal memory was the first variable to enter in the model and explained $35 \%$ of the 
Table 2: Results of Multiple Regression Analysis Examining the Contribution of Cognitive Variables to Predict Vineland Adaptive Behavior Scales (VABS) Domains Scores

\begin{tabular}{|c|c|c|c|c|c|}
\hline & $B$ & $S E$ & Standardized B & t-value & p-value \\
\hline \multicolumn{6}{|l|}{ Dependent variable $=$ Communication $^{a}$} \\
\hline $\mathrm{IQ}$ & 1.21 & 0.36 & 0.67 & 3.35 & 0.005 \\
\hline \multicolumn{6}{|l|}{ Dependent variable $=$ Daily living skills ${ }^{b}$} \\
\hline Verbal memory & 0.97 & 0.32 & 0.63 & 3.00 & 0.010 \\
\hline \multicolumn{6}{|c|}{ Dependent variable $=$ Socialization abilities ${ }^{c}$} \\
\hline Verbal memory & 1.09 & 0.49 & 0.45 & 2.25 & 0.042 \\
\hline $\mathrm{IQ}$ & 1.14 & 0.52 & 0.44 & 2.19 & 0.048 \\
\hline
\end{tabular}

${ }^{\mathrm{a}}$ Adjusted $\mathrm{R}^{2}=0.41, \mathrm{~F}=11.20, \mathrm{p}=0.005$

${ }^{\mathrm{b}}$ Adjusted $\mathrm{R}^{2}=0.35, \mathrm{~F}=9.01, \mathrm{p}=0.010$.

${ }^{c}$ Adjusted $R^{2}=0.49, F=8.06, p=0.005$.

variance (adjusted $R^{2}=0.346, F=8.94, p=0.010$ ). Final model included IQ, which increased the predictive value of the model an additional $14 \%$ (adjusted $R^{2}=$ $0.485, F=8.06, p=0.005$ ).

\section{DISCUSSION}

This cross-sectional study of cognition and adaptive functioning in children and adolescents with HF-ASD found a specific pattern of associations between verbal memory, IQ and adaptive functioning. As expected, IQ emerged as a main predictor of adaptive functioning. Also verbal memory emerged as a significant predictor of adaptive scores in our sample independently from $I Q$, highlighting the role of verbal memory as a predictor of adaptive functioning in HF-ASD.

Regarding adaptive domains, more than a third of the variance in Communication domain was predicted by IQ (41\%). Adaptive communication abilities have been previously found to be predicted by IQ $[9,23]$ and also by language and verbal memory tests [2]. In our sample, only IQ was significantly related with communication abilities and, contrary to Liss et al. [2] results, verbal memory was not associated with this domain. Also, it has been suggested that working memory might play an even stronger role in the VABS Communication domain [2]. Consistenly, OliverasRentas et al. [4] found that working memory was significantly correlated with the Communication domain of the VABS. Nonetheless, they used correlational analysis, so the specific contribution of working memory on communication abilities variance beyond IQ was not examined. In our results, working memory scores were not significantly related to the communication domain. We assessed the working memory domain through the Letter-Number
Sequencing subtest of the Wechsler scale. In the Oliveras-Rentas et al. [4] study the working memory score was a combination of the Letter-Number Sequencing subtest and the Digit span subtest although in some cases they used the Arithmetic subtest instead of the Letter-Number Sequencing subtest. These differences in the assessment strategy could be related with the differences in the results.

A significant proportion of the variance in Daily Living Skills was predicted by verbal memory (35\%) and almost half of the variance in Socialization domain was predicted by both, verbal memory and IQ (49\%). Our findings are consistent with Liss et al. [2] highlighting the strong impact of verbal memory performance on these adaptive skills in HF-ASD patients independently from IQ. Recently, others have noted that verbal memory in HF-ASD adolescents might be relevant to predict success in achieving autonomy [24]. Furthermore, because it has been shown that task support during memory tasks might improve ASD patients' performance [25], improvement on verbal memory might become a primary target in HF-ASD intervention programs.

Obviously, the present study has some important limitations. The small sample size has to be acknowledged. It is possible that some relationships between cognitive variables and adaptive functioning were underestimated due to low statistical power. Even though all patients fulfilled DSM-IV criteria for ASD, specific standardized instruments for autistic disorders were not used in the clinical assessment. Finally, the cross-sectional nature of the study has to be taken into account. Future longitudinal studies are needed to confirm the value of cognition as predictor of adaptive functioning in HF-ASD. Despite these limitations, the 
study also has strengths such as the examination of less studied cognitive functions and the use of regression methods in the analysis. It is also of relevance the accuracy of the assessment and administration of the neurocognitive battery performed by an expert clinician with extensive experience in child and adolescent.

In conclusion, this study adds a piece of evidence on the field of cognitive predictors different than IQ for adaptive behavior in HF-ASD. The evidence for a predictive relationship between some specific cognitive domains with adaptive functioning could improve our understanding of the links between them, and more importantly, could suggest new areas of intervention. While acknowledging that future studies are needed, this study highlights the role of verbal memory abilities for adaptive functioning in HF-ASD and informs about potential targets for intervention approaches.

\section{CONFLICT OF INTEREST}

The authors declare that they have no conflicts of interest.

\section{ROLE OF FUNDING SOURCE}

This study was supported in part by the Government of Catalonia, Comissionat per Universitats i Recerca del Departament d'Innovació, Universitats i Empresa (DIUE) 2009-SGR-1119. The DIUE had no further role in study design; in the collection, analysis and interpretation of data; in the writing of the report; and in the decision to submit the paper for publication.

\section{REFERENCES}

[1] Klin A, Saulnier CA, Sparrow SS, Cicchetti DV, Volkmar FR, Lord C. Social and communication abilities and disabilities in higher functioning individuals with autism spectrum disorders: the Vineland and the ADOS. J Autism Dev Disord 2007; 37: 748-59. http://dx.doi.org/10.1007/s10803-006-0229-4

[2] Liss M, Harel B, Fein D, et al. Predictors and correlates of adaptive functioning in children with developmental disorders. J Autism Dev Disord 2001b; 31: 219-30. http://dx.doi.org/10.1023/A:1010707417274

[3] Perry A, Flanagan HE, Dunn GJ, Freeman NL. Brief report: the Vineland Adaptive Behavior Scales in young children with autism spectrum disorders at different cognitive levels. J Autism Dev Disord 2009; 39: 1066-78. http://dx.doi.org/10.1007/s10803-009-0704-9

[4] Oliveras-Rentas RE, Kenworthy L, Roberson III RB, Martin A, Wallace GL. WISC-IV Profile in High-Functioning Autism Spectrum Disorders: Impaired Processing Speed is Associated with Increased Autism Communication Symptoms and Decreased Adaptive Communication Abilities. J Autism Dev Disord 2012; 42: 655-64. http://dx.doi.org/10.1007/s10803-011-1289-7
[5]

Chang C, Lung F, Yen C, Yang P. Adaptive Behaviors in High-Functioning Taiwanese Children with Autism Spectrum Disorders: an Investigation of the Mediating Roles of Symptom Severity and Cognitive Ability. J Autism Dev Disord 2013; 43: 1347-55.

http://dx.doi.org/10.1007/s10803-012-1684-8

[6] Kanne SM, Gerber AJ, Quirmbach LM, Sparrow SS, Cicchetti DV, Saulnier CA. The role of adaptive behavior in autism spectrum disorders: implications for functional outcome. J Autism Dev Disord 2011; 41: 1007-18. http://dx.doi.org/10.1007/s10803-010-1126-4

[7] Sparrow SS, Cicchetti DV, Balla A. Vineland Adaptive Behavior Scales. Circle Pines, MN: American Guidance Service 2005.

[8] Charman T, Jones C, Pickles A, Simonoff E, Happé F. Defining the cognitive phenotype of autism. Brain Res 2011; 1380: 10-21. http://dx.doi.org/10.1016/j.brainres.2010.10.075

[9] Kenworthy L, Case L, Harms MB, Martin A, Wallace GL. Adaptive behavior ratings correlate with symptomatology and IQ among individuals with high-functioning autism spectrum disorders. J Autism Dev Disord 2010; 40: 416-23. http://dx.doi.org/10.1007/s10803-009-0911-4

[10] Szatmari P, Merette C, Bryson SE, et al. Quantifying dimensions in autism: a factor-analytic study. J Am Acad Child Adolesc Psychiatry 2002; 41: 467-74. http://dx.doi.org/10.1097/00004583-200204000-00020

[11] Harrison PL, Oakland T. Adaptive behavior assessment system. 2nd ed. Taipei: The Chinese Behavioral Science Corporation (Chinese version) 2008.

[12] Gilotty L, Kenworthy L, Sirian L, Black DO, Wagner AE. Adaptive skills and executive functions in autism spectrum disorders. Child Neuropsychol 2002; 8: 241-48.

http://dx.doi.org/10.1076/chin.8.4.241.13504

[13] Gioia G, Isquith P, Guy S, Kenworthy L. BRIEF: Behavior Rating Inventory of Executive Function. Odessa, FL: Psychological Assessment Resources 2000.

[14] Ozonoff S, Cook I, Coon H, et al. Performance on Cambridge Neuropsychological Test Automated Battery subtests sensitive to frontal lobe function in people with autistic disorder: evidence from the Collaborative Programs of Excellence in Autism network. J Autism Dev Disord 2004; 34 139-50. http://dx.doi.org/10.1023/B:JADD.0000022605.81989.cc

[15] Liss $M$, Fein $D$, Allen $D$, et al. Executive functioning in highfunctioning children with autism. J Child Psychol Psychiatry 2001a; 42: 261-70.

http://dx.doi.org/10.1111/1469-7610.00717

[16] Pellicano E. The Development of Executive Function in Autism. Autism Res Treat 2012; 1-8.

\section{http://dx.doi.org/10.1155/2012/146132}

[17] Grinter EJ, Maybery MT, Pellicano E, Badcock JC, Badcock DR. Perception of shapes targeting local and global processes in autism spectrum disorders. J Child Psychol Psychiatry 2010; 51(6): 717-24.

http://dx.doi.org/10.1111/j.1469-7610.2009.02203.x

[18] American Psychiatric Association. Diagnosis and statistical manual of mental disorders. 4th ed. text revision. Washington DC: American Psychiatric Association 2000.

[19] Wechsler D. The Wechsler Intelligence Scale for Children (WISC-IV). Madrid: TEA Ediciones 2003.

[20] Reynolds CR, Bigler ED. Test of memory and learning (TOMAL). Austin, Texas: ProEd 1994.

[21] Rey A. Rey-Osterrieth Complex Figure Test. Madrid: TEA 1987.

[22] Heaton RK. Wisconsin Card Sorting Test Manual (WCST). Odessa, FL: Psychological Assessment Resources 1981. 
[23] Black DO, Wallace GL, Sokoloff JL, Kenworthy L. Brief Report: IQ split predicts social symptoms and communication abilities in high-functioning children with autism spectrum disorders. J Autism Dev Disord 2009; 39: 1613-19. http://dx.doi.org/10.1007/s10803-009-0795-3

[24] Jones CR, Happe F, Pickles A, et al. 'Everyday Memory' Impairments in Autism Spectrum Disorders. J Autism Dev Disord 2011; 41: 455-64.

http://dx.doi.org/10.1007/s10803-010-1067-y
[25] Phelan HL, Filliter JH, Johnson SA. Brief Report: Memory Performance on the California Verbal Learning Test Children's Version in Autism Spectrum Disorder. J Autism Dev Disord 2011; 41: 518-23.

http://dx.doi.org/10.1007/s10803-010-1069-9

Received on 09-07-2013

Accepted on 18-09-2013

Published on 28-10-2013

DOI: http://dx.doi.org/10.6000/2292-2598.2013.01.01.4

(C) 2013 Puig et al.; Licensee Lifescience Global.

This is an open access article licensed under the terms of the Creative Commons Attribution Non-Commercial License (http://creativecommons.org/licenses/by-nc/3.0/) which permits unrestricted, non-commercial use, distribution and reproduction in any medium, provided the work is properly cited. 